\title{
Application à la zone au large du Finistère d'une méthode d'estimation statistique des courants de houle au voisinage du fond
}

PAR

A. Cavanié

C.N.E.X.o.

\section{Introduction}

L'estimation des statistiques à long terme des courants dus à la houle près du fond sur le plateau continental, mais loin des côtes, est un problème qui préoccupe à juste titre l'ingénieur chargé d'opérations offshore. Très souvent, le coût et les délais impliqués par des mesures de longue durée interdisent d'aborder ce problème directement (il est rare qu'un ingénieur puisse attendre une réponse plus d'un an sans perdre patience!). Par ailleurs, l'utilisation d'observations de navires pour déduire de la houle en surface les mouvements sur le fond, bute sur le fait que les «périodes " et les hauteurs significatives signalées sont en fait des valeurs moyennes alors qu'il faudrait connaitre l'énergie aux plus longues périodes qui seules apportent une contribution notable aux courants sur le fond.

Parce que le modèle DSA 5 fournit des estimations de l'énergie dans la houle par bandes spectrales, il permet de passer du spectre des déplacements en surface au spectre des courants au fond, en appliquant, bande par bande, la fonction de transfert appropriée. C'est l'exposé de cette méthode et son application aux données du modèle de 1970 à 1974 au Point Bretagne $\left(47^{\circ} 30 \mathrm{~N}\right.$ et $\left.5^{\circ} \mathrm{W}\right)$ qui fait l'objet de cette communication. Nous tenons à remercier ici $M$. Gelci, Directeur de l'Antenne de la Météorologie Nationale au Centre Océanologique de Bretagne qui nous a communiqué l'ensemble des données du modèle que nous avons utilisé.

* Contribution $n^{\circ} 370$ du Département Scientifique du Centre Océanologique de Bretagne.
Conscient des limites des différentes approximations utilisées et cherchant surtout à fixer les ordres de grandeur des courants et leurs probabilités, nous n'avons tenu compte ni des gains, ni des pertes d'amplitude liés à la diminution de profondeur, au frottement sur le fond, à la réflexion ou à la réfraction. Dans l'étude d'un site spécifique, sur lequel seraient prévues des opérations importantes, un étalonnage du modèle par des mesures pendant une année permettrait de lever l'ensemble de ces hypothèses.

\section{1 - Les données du modèle DSA 5}

Le modèle DSA 5 de la Météorologie Nationale. qui prédit à partir du champ de vent les conditions de mer sur l'Atlantique Nord, fonctionne depuis plusieurs années [1] [2]. Étalonné au point $K$, il donne une juste idée des spectres de houle tant que l'influence du fond n'est pas prépondérante. A titre de comparaison avec des observations de navires, sont reportés sur la figure 1 les rapports (en \%) du nombre de prédictions du modèle dans différentes plages de hauteurs significatives, au nombre total (5 647) de prédictions s'étendant sur les quatre années de 1971 à 1974 (quatre mois de l'année 1970 ayant été utilisés pour remplir un vide équivalent en 1971); ceci représente presque l'ensemble de 5840 estimations effectuées quatre fois par jour pendant quatre ans (les quelques trous dans les mesures sont dus à des incidents techniques d'ordre divers) au point Bretagne situé à la position $47^{\circ} 30 \mathrm{~N}$ et $5^{\circ} \mathrm{W}$. Sur la même figure et de la même façon, sont reportées 46547 ob- 


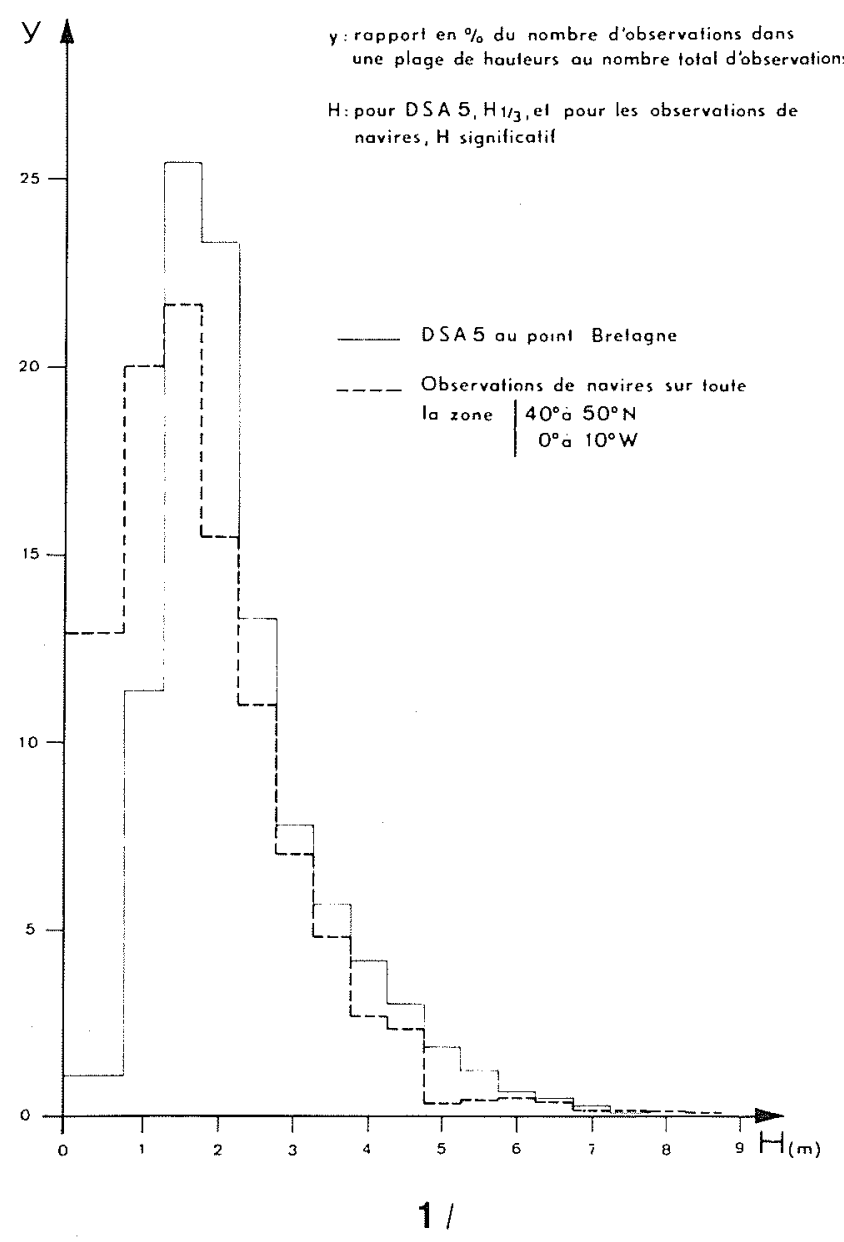

servations de navires effectuées entre 1953 et 1961 dans le carreau Marsden $40^{\circ}$ à $50^{\circ} \mathrm{N}, 0^{\circ}$ à $10^{\circ} \mathrm{W}$ relevées dans l'atlas d'Hogben et Lumb [3]. En raison de l'étendue de la zone d'observations, il n'est pas logique d'espérer un accord parfait entre des deux sources de renseignement. Le modèle prévoit beaucoup moins de petites valeurs de $H_{1 / 3}$ que les observations, ce qui peut être dû à la fois à la situation au large du Finistère et aux observateurs qui rapportent souvent des valeurs sous-estimées de la houle lorsqu'elle est très longue et donc de faible cambrure.

\section{2 - Application des données du modèle DSA 5 à l'estimation des courants sur le fond}

Les données sur fichier à la Météorologie Nationale comprennent, quatre fois par jour, une estimation de l'énergie dans six plages de périodes, larges chacune de trois secondes et qui sont centrées sur $4,7,10,13,16$ et 19 secondes.

Pour une onde de gravité de petite amplitude ayant pour équation de la surface libre

$$
\eta(x, t)=A \cos (k x-\omega t)
$$

la vitesse horizontale $u$ à une profondeur $z$ sera donnée par l'équation:

où

$$
u=U \cos (k x-\omega t)
$$

$$
U=[\omega A \cosh k(z+D)] / \sinh k D
$$

Au voisinage du fond, $U$ sera donnée (en dehors de la couche limite, ici négligée) par :

$$
U_{n}=2 \pi A /[T \sinh (2 \pi D / L)]
$$

Les longueurs d'ondes $L$ sont données implicitement en fonction des périodes $T$ et des profondeurs $D$ par l'équation :

$$
2 \pi /\left(g T^{\prime \prime}\right)=[\tanh (2 \pi D / L)] / L
$$

Pour une profondeur $D$ de cent mètres, seule considérée ici. le tableau suivant donne les valeurs calculées de $L$ en fonction des périodes moyennes, $T$. des bandes du modèle DSA 5 .

\begin{tabular}{|c|c|}
\hline$T$ & $L$ \\
$(\mathrm{~s})$ & $(\mathrm{m})$ \\
\hline 4 & 24,95 \\
\hline 7 & 76,43 \\
\hline 10 & 155.87 \\
\hline 13 & 259,47 \\
\hline 16 & 372,77 \\
\hline 19 & 484,67 \\
\hline
\end{tabular}

Il suffit maintenant de multiplier l'énergie dans chaque bande du modèle par la valeur de $\left(U_{n} / A\right)^{-2}$ correspondant à la période moyenne de cette houle :

\begin{tabular}{|c|c|}
\hline$T$ & $\left(U_{v} / A\right)^{2}$ \\
\hline 4 & négligeables \\
\hline 7 & négligeables \\
\hline 10 & $4.9817 .10^{-4}$ \\
\hline 13 & $7.4831 .10^{--3}$ \\
\hline 16 & $2.2725 .10^{-\cdots}$ \\
\hline 19 & $3.8232 .10^{-2}$ \\
\hline
\end{tabular}

pour trouver l'énergie du spectre des courants dans cette bande de périodes au fond. 


\section{3 - Étude des statistiques à long terme des courants par $100 \mathrm{~m}$ de fond}

Pour un spectre étroit de houle $\Phi_{\eta}(T)$ Cartwight et Longuet Higgins ont montré que la valeur de $H_{1 / 3}$ est donnée par l'équation

$$
H_{1 / 3}=4\left[\int_{-11}^{\infty} \Phi_{\eta}(T) d T\right]^{1 / 2}
$$

De même. compte tenu du fait que seules les composantes du spectre de basses fréquences atteignent le fond (ce qui assure l'étroitesse du spectre) l'on peut définir $U_{1 / 3}$, en prenant non plus les variations "crête-à-creux " mais les valeurs des maximums ou des minimums du courant, ce qui conduit à l'équation :

$$
H_{1 / 3}=2\left[\int_{0}^{\infty} \Phi_{11}(T) d T\right]^{1 / 2}
$$

L'ensemble des valeurs de $U_{1 / 3}$ ainsi calculées est reporté sur la figure 2 sur un papier de Weibull; en abscisse $U_{1 / 3}$. en ordonnée la probabilité cumulative $P\left(U_{1 / 3}\right)$, qui, pour une loi de Weibull doit satisfaire l'équation :

$$
P\left(U_{1 / 3}\right)=1-\exp ^{-\mid \Gamma_{1 / 3} / A / r}
$$

où $A$ et $c$ sont des constantes appropriées

L'accord avec la loi de Weibull paraît très satisfaisant. les points expérimentaux s'écartent très peu de la droite correspondant à cette loi. D'après la figure 2, si dans $50 \%$ des cas, les valeurs de $U_{1: 3}$ ne dépassent pas sensiblement $5 \mathrm{~cm} / \mathrm{s}$, des valeurs de $40 \mathrm{~cm} / \mathrm{s}$ sont atteintes dans $1 \%$ des cas de $60 \mathrm{~cm} / \mathrm{s}$ dans $0,1 \%$ des cas.

\section{Conclusions}

La plupart du temps, l'importance des courants de houle par $100 \mathrm{~m}$ est, d'après les calculs exposés, relativement faible par rapport aux courants de marée de l'ordre du nœud dans cette zone. Mais pendant quelques dizaines d'heures en moyenne par an, les courants de houle, superposés aux courants de marée, peuvent jouer un rôle non négligeable dans les processus sédimentaires ou engendrer des efforts alternatifs notables dans les structures artificielles au fond (réservoirs, pipes-lines, etc.).

Plusieurs problèmes restent posés, qu'il faudra à lavenir préciser : en particulier l'influence de la réfraction et de la remontée des fonds, ainsi que l'étalonnage du modèle par des mesures de courant de houle au voisinage du fond. Ces travaux apporteront certainement une plus grande préci-
PROBABILITE CUMULAIIVE $P\left(U_{1 / 3}\right)$ EN FONCTION DE $U_{1 / 3}$

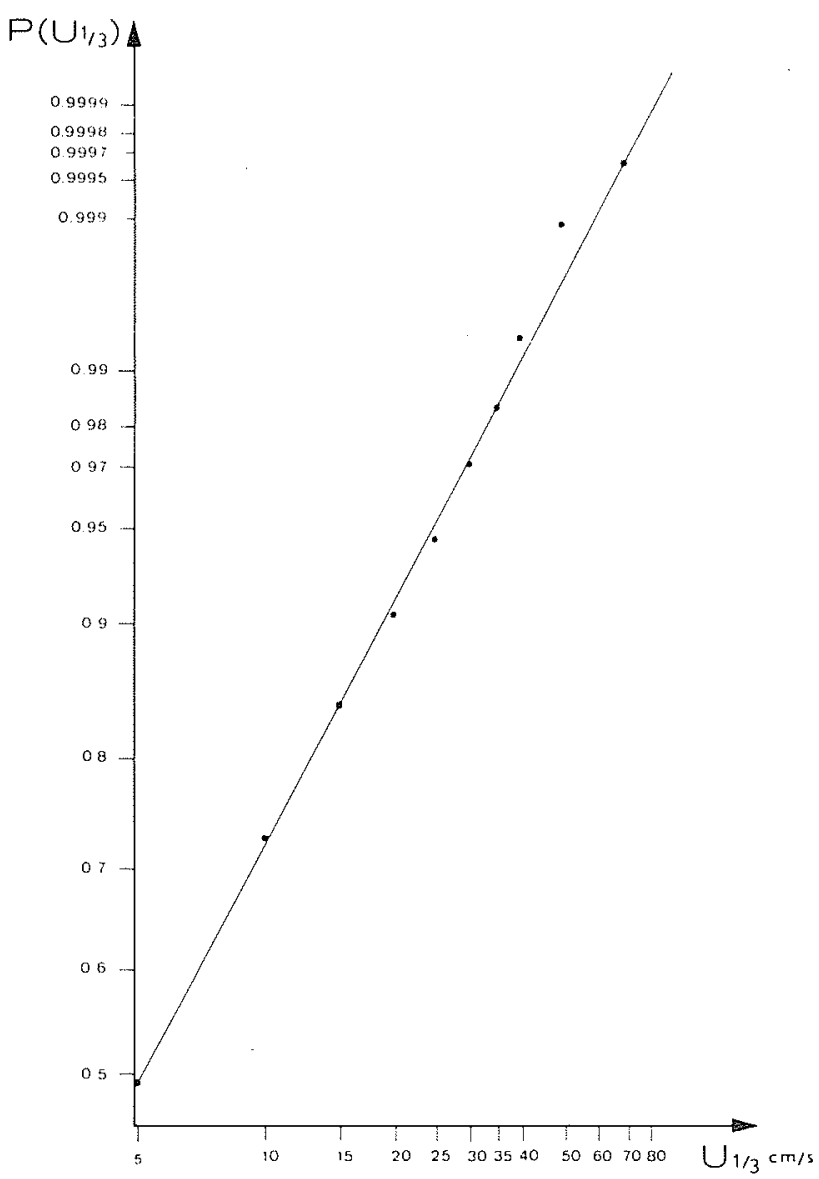

2 / Probabilité cumulative $P_{\left(U_{1 / 3}\right)}$ en fonction de $U_{1 / 3}$

sion dans nos estimations. mais les conclusions générales tirées de létude des données du modèle DSA 5 resteront certainement valables.

\section{Bibliographie}

[1] Stvist (A.) et Foxs (C.), - Analyse et prévision de l'état de la mer: méthode DSA 5. La Homille Blanche, n" 31966. pp. 321-337

[2] Geicl (R.) et Drviliez (E.). - Le calcul numérique de l'état de la mer. La Houlle Blatche. n" 2 1970. pp. 117-136.

[3] Hoghex (N.) et LUMB (F.). - "Ocean wave statistics" London. H.M.S.O. (1967). 


\section{Discussion}

Président: M. le Professeur LACOMBE

M. le Président remercie M. Cavanié de son intéressant exposé qui donne une estimation de la distribution du tiers maximal des vitesses de houle - au large du Finistère - au voisinage d'un fond à $100 \mathrm{~m}$ sous la surface libre; et ce, par application de la théorie linéaire classique de la houle. C'est là un résultat important car on s'intéresse beaucoup actuellement à la mer d'Iroise.

Nous avons constaté avec satisfaction que les résultats des DSAs sont assez bons et noté que le réseau d'enregistrement, en cours d'installation dans la région, permettra bientôt de les tester de façon plus approfondie.

Le modèle global, précise M. CAVANIÉ, tient compte de 18 directions par tranche de période. Mais, considérant que seules les composantes à 13,16 et 19 secondes interviennent de façon significative dans la production de courant sur le fond et compte tenu d'autres observations océanographiques, qui montrent que le spectre directionnel est d'autant plus étroit que la période est longue, j'ai fait l'hypothèse qu'on pouvait concentrer toute cette énergie dans une même direction.

Je suppose, remarque $M$. le Président, que les vitesses que vous avez calculées - elles atteignent $0,80 \mathrm{~m} / \mathrm{s}$ pendant une douzaine d'heures par an - sont susceptibles, superposées aux courants de marée, de mettre en mouvement des matériaux fins sur le fond. Cela expliquerait les phénomènes importants de balayage des fonds signalés dans la première communication de cette séance.

Répondant à M. Lepetit, M. CAVANiÉ confirme que, bien que la méthode DSA fournisse des spectres multidirectionnels, le $U_{1 / 3}$ qu'il a calculé correspond à l'énergie globale dans toutes les directions.

Personne ne demandant la parole, M. le Président lève la séance en remerciant, à nouveau, les auteurs des communications et tous ceux qui sont intervenus dans leurs discussions.

\section{Abstract \\ Application to an area off the Finistere coast of a statistical method of estimating wave currents near the sea bed}

The spectral energy distribution of sea waves is supplied four times a day by the French Meteorological Office's DSA: model using the pressure distribution over the Atlantic as input. The surface-motion spectrum furnished by the model may then be used to estimate the local current spectrum near the sea bed. The present study is based on four years (1970-1974) of DSAs data for the so-called Brittany point $\left(47^{\circ} 30 \mathrm{~N}, 5^{\prime \prime} \mathrm{W}\right)$. The data correlates reasonably well with observations made from ships in the region lying between $40^{\circ}-50^{\circ} \mathrm{N}$ and $0^{\circ}-10^{\circ} \mathrm{W}$ (Figure 1).

Plotted on Weibull paper in Figure 2 are the cumulative probability values of $U_{1 / 3}$ as calculated from the DSAs data
( $U_{1 / 3}$ is the mean of the upper third of maximum current values at a depth of $100 \mathrm{~m}$ ). The experimental $U_{1 / 3}$ statistics appear to obey Weibull's law quite closely. They also show that $0.7 \mathrm{~m} / \mathrm{s}$ currents occur on average once a year.

The principal objective of the study was to provide orderof-magnitude estimates of currents. Accordingly, factors such as reflection, diffraction, bed friction, and loss or gain of amplitude due to reduced depth were neglected. The extent to which this is justified might be assessed by carrying out model calibration tests in an area of particular interest in the course of one winter. 